日本原子力研究開発機構機関リポジトリ

Japan Atomic Energy Agency Institutional Repository

\begin{tabular}{|c|l|}
\hline Title & $\begin{array}{l}\text { Cavitation erosion induced by proton beam bombarding mercury } \\
\text { target for high-power spallation neutron sources }\end{array}$ \\
\hline Author(s) & $\begin{array}{l}\text { Futakawa Masatoshi, Naoe Takashi, Kogawa Hiroyuki, } \\
\text { Haga Katsuhiro, Okita Kohei }\end{array}$ \\
\hline Citation & Experimental Thermal and Fluid Science,57,p.365-370 \\
\hline Text Version & Accepted Manuscript \\
\hline URL & $\underline{\text { https://jopss.jaea.go.jp/search/servlet/search?5046620 }}$ \\
\hline DOI & $\underline{\text { https://doi.org/10.1016/j.expthermflusci.2014.05.014 }}$ \\
\hline Right & $\begin{array}{l}\text { C 2014. This manuscript version is made available under the } \\
\text { CC-BY-NC-ND 4.0 license } \\
\text { http://creativecommons.org/licenses/by-nc-nd/4.0/ }\end{array}$ \\
\hline
\end{tabular}




\title{
Cavitation erosion induced by proton beam bombarding mercury target for high-power spallation neutron source
}

\author{
Masatoshi Futakawa ${ }^{a}$, Takashi Naoe ${ }^{a}$, Hiroyuki Kogawa ${ }^{a}$, Katsuhiro Haga ${ }^{a}$, \\ Kohei Okita ${ }^{b}$ \\ a Japan Atomic Energy Agency, Tokai-mura, Naka-gun, Ibaraki 319-1195, Japan. \\ ${ }^{b}$ College of Industrial Technology, Nihon University, Narashino, Chiba 275-8575, Japan.
}

\begin{abstract}
A liquid mercury target system for a megawatt-class spallation neutron source is being developed in the world. Proton beam is injected to the mercury target to induce spallation reaction. The moment the proton beams bombard the target, pressure waves are generated in the mercury by the thermally shocked heat deposition. The pressure waves excite the mercury target vessel and negative pressure that may cause cavitation along the vessel wall. Gas-bubbles will be injected into the flowing mercury to mitigate the pressure waves and suppress the cavitation inception. The injected gas-bubbles conditions were examined and the effects were predicted experimentally and theoretically from the viewpoints of macroscopic time-scale and microscopic time-scale, i.e. in the former is dominant the interaction between the structural vibration and the pressure in mercury, and in the later is essential the pressure wave propagation process.

Keywords: Pressure waves, Thermal shock, Proton beam, Spallation neutron source, Cavitation, Microbubbles, Liquqid metal
\end{abstract}

Email address: futakawa.masatoshi@jaea.go.jp (Masatoshi Futakawa) 


\section{Introduction}

Neutrons are used for the innovative research that will bring about breakthrough in scientific and engineering research fields, i.e. fuel cell, hydrogen embrittlement, protein structure, medicine, etc. Mercury has the benefits for pulse spallation neutron sources because of the high neutron yielding efficiency and usage as a coolant, and is available as target material to produce neutrons by spallation reaction that is caused by the bombardment in mercury with high-energy protons. The pulsed spallation neutron sources are being operated at the Material and Life science experimental Facility (MLF) in the Japan Proton Accelerator Research Complex (J-PARC) in Japan [1] and the Spallation Neutron Source (SNS) in the USA [2], which are standing on the way to increase the power up to megawatt-class. However, the higher the proton beam power, the severer the mercury cavitation problems become apparent.

At the moment that the proton beam bombards in the mercury, thermal shock is generated in the mercury and the pressure waves are induced [3-5], whose amplitude is dependent on the proton beam power. On the process of the pressure wave propagation aggressive cavitation generates in the mercury, which imposes damage on the solid wall of the target vessel. Therefore, the cavitation phenomenon gets to be a crucial issue to increase the power in the mercury target for the pulsed spallation neutron sources, which is illustrated in Fig. 1. Theoretical and experimental investigations were carried out to understand the relationships among pressure wave conditions, mercury cavitation aggressiveness, and damage growth behaviors on some solid materials $[6,7]$.

Surface hardening treatments of the target vessel wall by coatings and/or surface improvements, Kolestrising ${ }^{\circledR}$, nitriding, etc., were tried to reduce the cavita- 
tion damage formation $[8,9]$. These techniques were not sufficient to protect the vessel wall against damages due to very violent mercury cavitation. On the other hand, microbubble injection for softening mercury is investigated from the viewpoint of pressure wave mitigation. However, such technique is not straightforward because of complicated phenomenon on interaction between injected gas bubbles and cavitation bubbles under dynamic pressure responses, which are very dependent on the interaction between the solid vessel wall and the impulsive pressure induced by protons injection. As well, it was not easy to make the microbubbles in flowing mercury which are theoretically predicted to mitigate the pressure: i.e. the

diameter of bubbles is less than $100 \mu \mathrm{m}$ and the void fraction larger than $10^{-3}$ [10], because mercury exhibits high surface tension, high density, low wettability, etc. An innovative microbubble generator, therefore, a so-called swirl bubbler was developed to inject microbubbles into flowing mercury [11]. However, the bubbling condition is not sufficient as compared with the theoretical prediction.

In this paper, the gas-bubbling mitigation effects are discussed based on the experimental observation and the numerical simulation on the interaction between the gas-bubbles and the cavitation bubbles.

\section{Pressure waves in mercury target}

\subsection{Rapid thermal expansion by proton injection}

Figure 1 shows the target vessel for the spallation neutron source at the MLF. The target vessel is filled with the mercury circulating at approximately $1 \mathrm{~m} / \mathrm{s}$ as flowing along the beam window. The spallation reaction is induced when accelerated protons ( $1 \mathrm{MW}, 3 \mathrm{GeV} 25 \mathrm{~Hz}$, and $1 \mu$ s pulse duration) are bombarded in mercury and approximately a half of the power is dissipated for rapidly heating in 


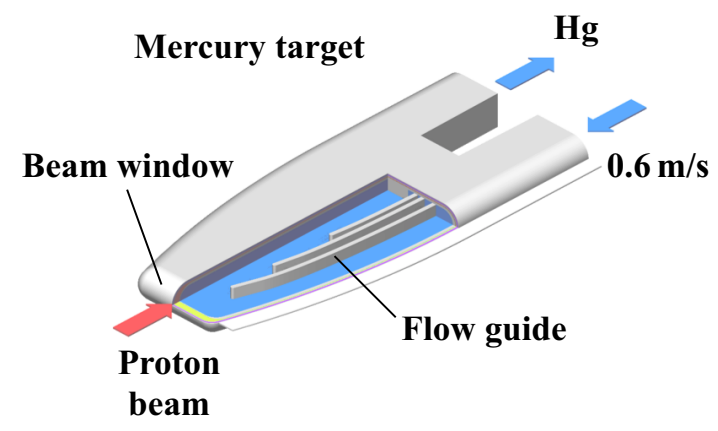

Figure 1: Mercury target at J-PARC. The drawing illustrates the inner wall of target vessel: i.e. the vessel consists of multi-wall structure to protect from the mercury leakage into the outside of vessel.

the mercury $[3,4,12]$. Temperature rising $\Delta T$ in the mercury is given by

$$
\Delta T=\frac{\Delta Q}{\rho C_{V}}
$$

where $T$ is the temperature, $Q$ is the heat density, $C_{V}$ is the specific heat capacity, $\rho$ is the density of mercury. As a result, pressure rapidly rises following the pressure waves in the mercury. Pressure rising $\Delta T$ in the mercury is given by

$$
\Delta P=\beta_{p} K_{T} \Delta T
$$

where $P$ is the pressure, $\beta_{p}$ is the thermal expansion, $K_{T}$ is the bulk modulus. Nuclear heat generation of the JSNS was calculated with the Particle and Heavy Ion Transport Code System (PHITS) [13] using the cross section data of Japanese Evaluated Nuclear Data Library (JENDL) Ver. 3.2. Nuclear heat distribution are very dependent on proton beam profiles. The maximum nuclear heat density $\left(Q_{\max }\right)$ in the mercury is associated with the proton current density: $Q_{\max }$ is the liner function of the proton current density. As assumed Gaussian distribution at $1 \mathrm{MW}$ proton beam, the maximum heat density is approximately $12 \mathrm{~J} / \mathrm{cc}$ with $1 \mu \mathrm{s}$ pulse duration. The maximum pressure become approximately $30 \mathrm{MPa}$. 


\subsection{Macroscopic time-scale}

Nuclear heat distributions $\Delta Q$ in the mercury target is very dependent on the proton beam profiles and the maximum peak pressure attributes to a peak heat deposition of the beam profile. In order to investigate the pressure propagation and the dependency of pressure waves on the beam profiles, FEM analyses were carried out using an explicit code LS-DYNA. The 1/4 symmetrical model was used for the FEM. Mercury and stainless steel vessel were meshed as solid and shell elements, respectively. The cut-off pressure model is applied to mercury to simulate the tension failure in mercury due to cavitation [14]. In the cut-off pressure model, a relationship between volumetric strain and pressure is linear elastic when pressure larger than a certain value (the cut-off pressure), but the mercury has no stiffness when the pressure is less than it. We used the cut-off pressure of $-0.15 \mathrm{MPa}$, whose suitability was confirmed experimentally. The boundary condition along the interface between solid wall and liquid mercury was tied. Pressure distribution in mercury due to the thermal expansion resulting from the proton beam injection was applied for the initial condition.

Figure 2 shows the pressure time response nearby the beam window in liquid mercury along the center axis in the proton beam injection direction under $1 \mathrm{MW}$ power. After the strong compressive pressure appears, the long period of negative pressure is $5 \mathrm{~ms}$ approximately, and the pressure increases gradually after around $8 \mathrm{~ms}$. The negative pressure with a relatively long period is induced by the interaction between the solid wall and the liquid mercury.

The damage is dependent on the cavitation bubble growth and collapse behaviors, i.e. cavitation intensity, which are affected by the amplitude and the timeresponse of pressure waves. In the macroscopic view, it can be said that the liquid 


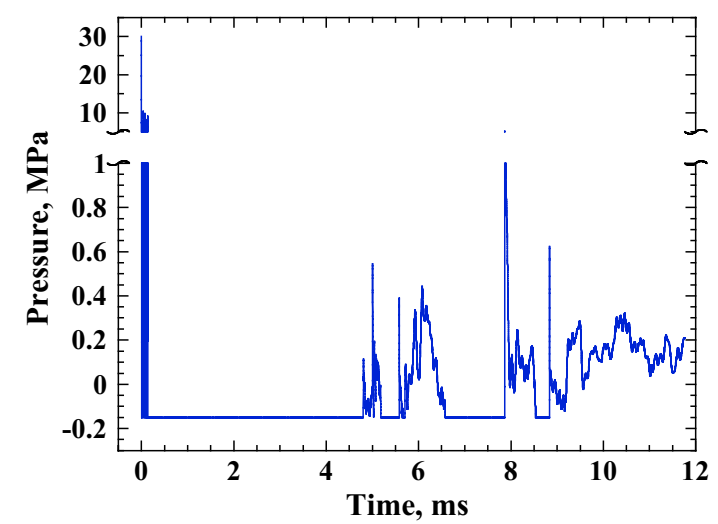

Figure 2: Time-response of pressure waves nearby mercury target.

failed at the pressure lower than a critical tensile pressure to induce the cavitation bubbles, i.e. so-called the Blake threshold, which is equivalent to the cut-off pressure used in the pressure wave propagation analysis in the target [14]. The period of negative pressure, therefore, seems to be one of crucial factors related with the cavitation intensity. The relationship between bubble growth behavior and pressure waves was investigated by using Rayleigh-Plesset equation The results showed that the cavitation inception is very dependent on the amplitude of negative pressure, and if the negative pressure exceeds the threshold, the maximum bubble size increases with the amplitude and imposing period of negative pressure.

\subsection{Microscopic time-scale}

The strong compressive pressure waves propagate toward the beam window from the highest heat deposition area around $30 \mathrm{~mm}$ in downstream of the beam injection direction with sound velocity in mercury. The compressive pressure waves are reflected and the phase might be changed by the acoustic impedance difference between the wall and the mercury so that the negative pressure oc- 
curs. Figure 3 shows the microscopic time-scale model, Pressure wave propagation Analysis Code in Mercury Target (PAC-MT) [10], to evaluate the effects of acoustic impedance of solid wall and the injected gas-bubbles, which is expected as one of the mitigation techniques to suppress the cavitation damage. The model is one dimensional axisymmetric propagation model with a rigid and an elastic wall and a heated area nearby the wall, which simulated the beam window of the target vessel. The energy conservation equation, the equation of state for liquid phase and the compressibility of liquid are considered. In the case of the gas liquid bubbly mixture, the heat transfer between the gas inside interface is considered to clarify the thermal damping effect for the bubble oscillation during the pressure propagation through the bubbly liquid. The gas inside bubbles is assumed to be noncondensable. The mass transfer through the gas-liquid interface such as evaporation, condensation and solution is not taken account. As for the elastic wall, the stiffness and the density were chosen to be equivalent to the beam window of $2.5 \mathrm{~mm}$ in thickness. The detailed model description is seen in Ref. [10].

Figure 4 shows the dependence of pressure wave on the wall stiffness. The strong compressive pressure is caused in the rigid boundary and the negative pressure is induced by the deflection of the elastic wall. In this study, in order to investigate the negative pressure which causes cavitation damage, the wall was treated as the elastic wall. The effect of injected gas-bubbles on the pressure waves will be discussed in the next section.

\section{Cavitation mitigated by gas bubbles}

We are trying to inject microbubbles into the mercury to reduce the pressure waves and suppress the cavitation inception $[10,15]$. The initial compressive pres- 


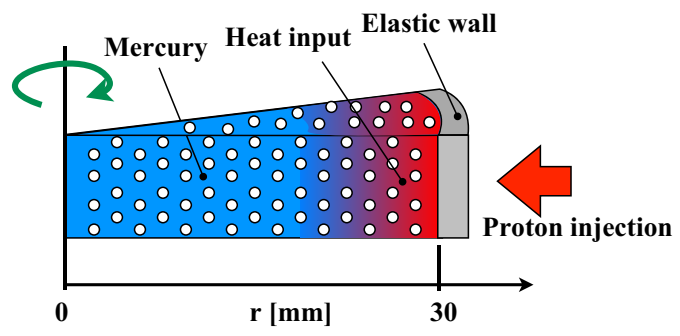

Figure 3: Microscopic time-scale model. One dimensional axisymmetric propagation model in the PAC-MT.

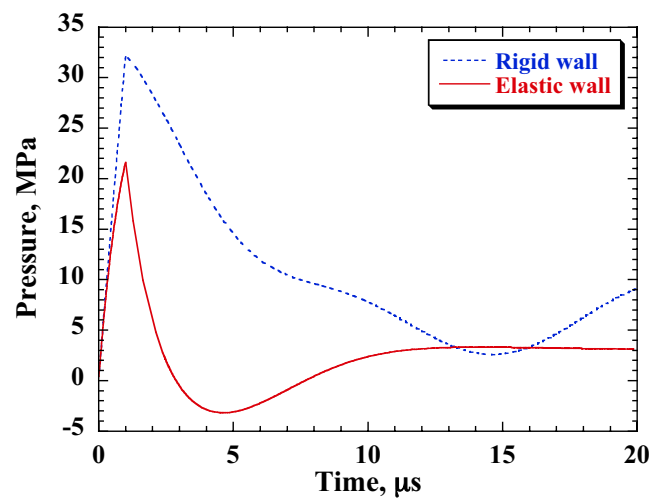

Figure 4: Elastic wall effect on pressure wave propagation. 
sure wave is reduced by absorption of the thermal expansion of mercury due to the contraction of microbubbles. The effect is expected at the heat deposited area and at the onset of pressure wave propagation. During the wave propagation, the microbubbles can reduce the amplitude of the compressive pressure waves through attenuation of the pressure waves due to the thermal dissipation of kinetic energy and the dispersion. The cavitation inception needs a certain negative pressure to grow the cavitation bubbles, i.e. so-called the Blake threshold. As assuming that the cavitation intensity is associated to the bubble size, the negative pressure magnitude and period are essential factor to determine the damage. It is deduced numerically that the magnitude and period resulting from the interaction between the intensive compressive pressure and the vessel wall and the inertia effect followed by the propagation of intensive compressive pressure waves are dependent on the beam power. The effects of microbubbles on the pressure waves and cavitation inception are conveniently categorized into macroscopic and microscopic time-scale-events: i.e. ms and $\mu$ s order events, as the followings.

\subsection{Macroscopic time-scale effects}

The pressure time-responses with ms-scale order are induced by the pressure wave propagation interacted with the vessel wall. Experimentally, the effect of gas bubbles was investigated by using electrical magnetic impact testing machine, MIMTM, which can impose compressive pressure on mercury through the strike disk of $100 \mathrm{~mm}$ in diameter [6, 15]. Figure 5 shows the schematic illustration of the mercury experimental loop. Helium gas-bubbles were injected through a porous tungsten bubbler from about $0.5 \mathrm{~m}$ upstream part under the ratio of the gas

flow rate to the mercury flow rate, controlled to be $10^{-3}$. The mercury flowing velocity in the flow channel of $33 \mathrm{~mm}$ in width and $12 \mathrm{~mm}$ in height is $0.3 \mathrm{~m} / \mathrm{s}$. 
The mean radius of gas bubbles ranges from $50 \mu \mathrm{m}$ to $100 \mu \mathrm{m}$. Figure 6 shows the pressure time-responses measured at the upper lid of the MIMTM under the stagnant and the flowing with injected gas-bubbles. The negative pressures followed the impulsive compressive pressures. The peak value of compressive pressure was hardly affected by the mercury conditions. On the other hand, the negative pressure was slightly changed by the conditions: i.e. in the case of the stagnant, the negative pressure is saturated at $-0.15 \mathrm{MPa}$ approximately, which is almost equivalent to the cut-off pressure used in the macroscopic time-scale model of LS-DYNA to describe the cavitation behavior, and the gas-bubbles with 50 100 $\mu \mathrm{m}$ in radius at void fraction of $10^{-4} \sim 10^{-3}$ have worked to slightly increase the negative pressure in the relatively long time-range pressure response.

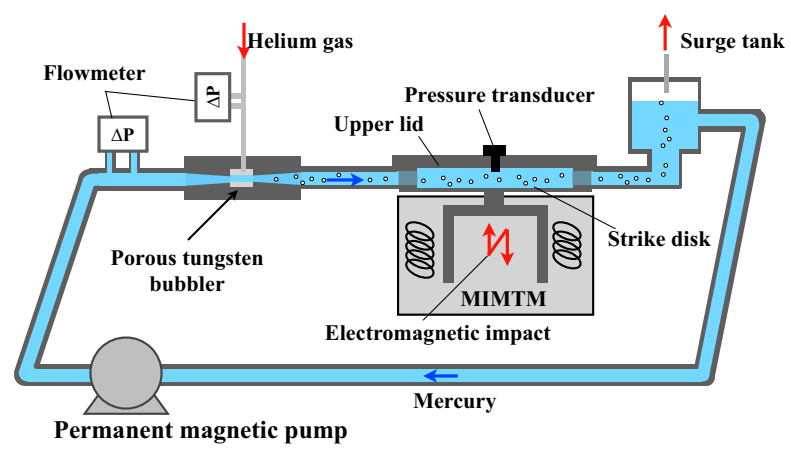

Figure 5: Schematic illustration of the mercury experimental loop. The MIMTM test section is located downstream of the bubbler. The upper lid of the MIMTM cylinder used in pressure measurement was replaced with a glass window for the bubble observation, and with a circular plate specimen for the damage test, respectively.

The cavitation inception and its bubble growth are very dependent on the negative pressure amplitude and imposing period. In order to evaluate the effect of gas bubbles on the cavitation inception, the cavitation bubble growth was predicted 


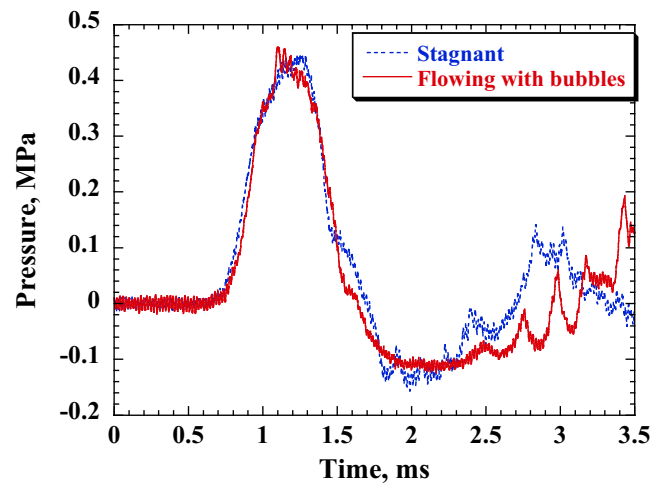

Figure 6: Pressure time-responses measured at MIMTM under stagnant and flowing with bubbles.

by using the Keller equation [16] as following:

$$
\begin{aligned}
& \left(1-\frac{\dot{R}}{C_{L}}\right) R \ddot{R}+\left(\frac{3}{2}-\frac{\dot{R}}{2 C_{L}}\right) \dot{R}^{2} \\
& =\frac{1}{\rho}\left(1+\frac{\dot{R}}{C_{L}}\right)\left(P_{b}[t]-P_{e x}\left[t+R / C_{L}\right]-P_{0}\right)+\frac{R}{\rho C_{L}} \dot{P}_{b}[t]
\end{aligned}
$$

where $R=R[t]$ is the time-dependent bubble radius, $R_{0}$ is an initial bubble radius (here, $R_{0}=20 \mu \mathrm{m}$ ), $C_{L}$ is the sound speed of liquid, $\rho$ is the density of liquid, $P_{e x}$ is the time-dependent external pressure which was given by the measured pressure under mercury condition, subscript 0 denotes the initial state, and overdots are the time derivative, respectively. $P_{b}$ and $P_{g}$ are the pressure on the liquid side and gas side of the bubble's interface as follows;

$$
\begin{aligned}
& P_{b}[t]=P_{g}[t]-\frac{2 \sigma+4 \eta \dot{R}[t]}{R[t]} \\
& P_{g}[t]=\left(P_{0}-P_{V}+\frac{2 \sigma}{R[t]}\right)\left(\frac{R_{0}}{R[t]}\right)^{3 \kappa}+P_{V}
\end{aligned}
$$

where $\sigma$ is the surface tension, $\eta$ the viscosity, $P_{V}$ the vapor pressure, respectively. The cavitation bubble growth behaviors for the pressure responses measured for stagnant and gas-bubbling conditions in Fig. 6 were calculated as shown in Fig. 7. 
The cavitation bubble grew under stagnant condition, on the other hand the cavitation hardly did. It can be said that the cavitation inception was sufficiently suppressed by the injected gas-bubbles. One possible reason for the suppression of cavitation inception is the compressive pressure waves emitted by expanding gas bubbles are superimposed on the negative pressure wave around cavitation nuclei and reduce the amplitude to a value lower than the threshold. This means that the compressive pressure emitted from gas bubbles suppresses cavitation inception [15].

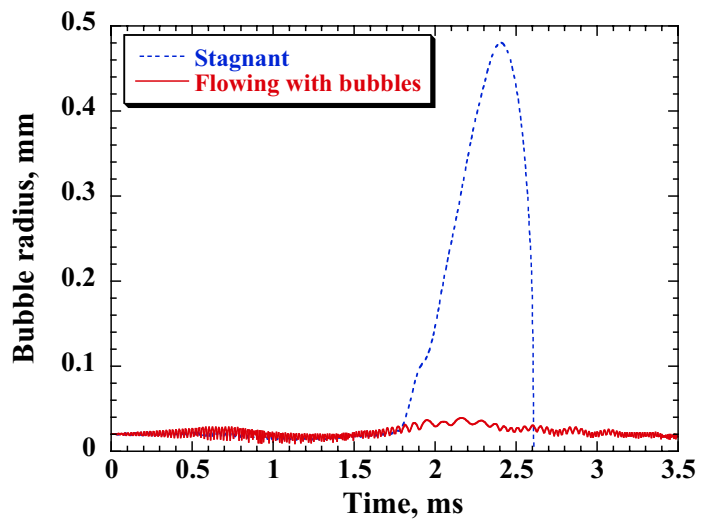

Figure 7: Bubble growth and collapsing behavior calculated using the Keller equation using the pressure responses shown in Fig. 6. $\left(R_{0}=20 \mu \mathrm{m}\right)$

Figure 8 (a) and (b) shows the typical photographic sequences of bubble collapsing behavior under both conditions, which were made from the captured image sequence obtained from high-speed video camera. More precisely, the upper lid of the MIMTM experimental loop was replaced to the rid with a glass window, bubbles behavior in contact with the glass window which were excited by the impulsive pressure were captured using the high-speed video camera. Then, the slice line of 1pixel in width passing through the bubble center was extracted 
from the each frame of captured image of $1.05 \times 10^{5} \mathrm{f} / \mathrm{s}$ in frame rate, and bound in order of time. The changes in radius in various bubbles measured from the images were shown in Fig. 8 (c) and (d). It can be seen that the collapsing behavior of gas-bubble is much milder than that in cavitation bubbles occurred in stagnant condition. As predicted in Fig. 7, the cavitation inception was not recognized optically under the flowing with gas-bubbles. Figure 9 shows the damaged plate fixedly-mounted on the upper lid of the MIMTM under both conditions: stagnant and flowing with gas-bubbles. The number of imposed impulsive pressures is $10^{4}$ cycles. The damage was hardly observed on the specimen under the gas-bubble condition, as compared with that under stagnant condition. We can understand well from Figs. 6 to 9, the gas bubbles with $50 \sim 100 \mu \mathrm{m}$ in radius with $10^{-3} \sim$ $10^{-4}$ void fraction are sufficiently workable for mitigating cavitation damage in macroscopic time-scale.

\subsection{Microscopic time-scale effect}

In the early stage within $10 \mu$ s after proton beam bombarding in mercury, a compressive pressure wave reaches the beam window and a negative pressure is induced by the phase change of compressive wave. Numerically was investigated the effects of injected microbubbles on the pressure wave propagation process by using PAC-MT. Figure 10 shows the effects of gas-bubbles with $50 \mu \mathrm{m}$ in radius at various void fractions on pressure time-responses. The mitigating effects by gas-bubbles are likely to be dependent on the void fractions. The compressive

pressure was hardly affected by $50 \mu \mathrm{m}$ gas-bubbles except for a relatively high void fraction of $5 \times 10^{-4}$, while the period of negative pressures is reduced as increasing the void fraction.

The effect of microbubble injection on the cavitation bubble growth was in- 


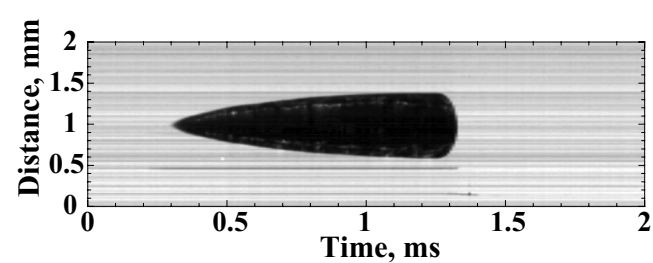

(a) Cavitation bubble growth under stagnant

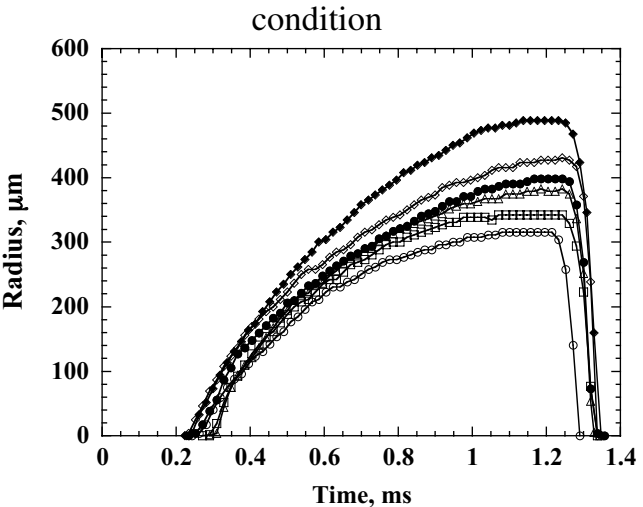

(c) Measured radius of cavitation bubbles

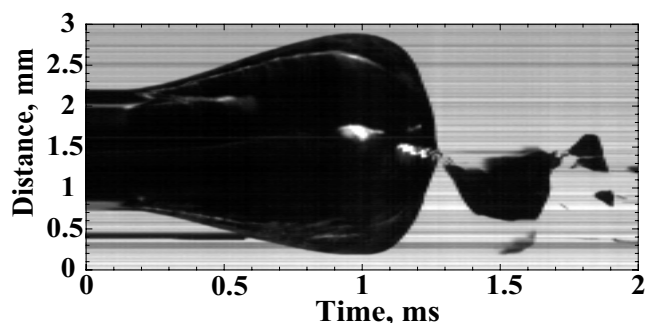

(b) Gas-bubble growth

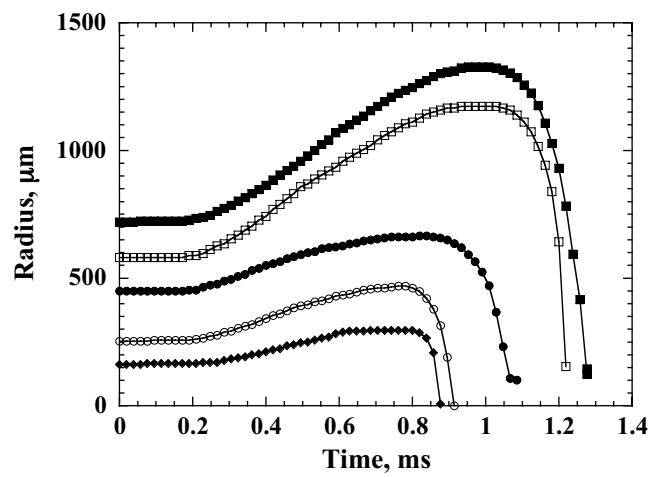

(d) Measured radius of gas-bubbles

Figure 8: Cavitation and gas-bubble growth behaviors.

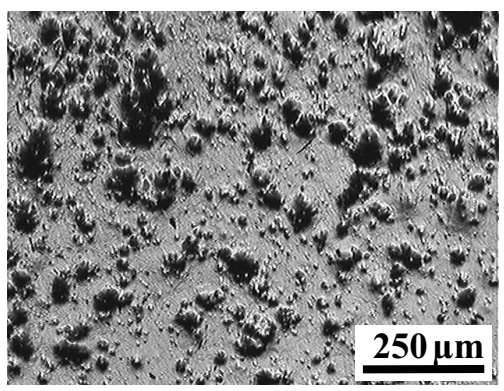

(a) Stagnant $\left(A_{e} / A_{0}=0.3\right)$

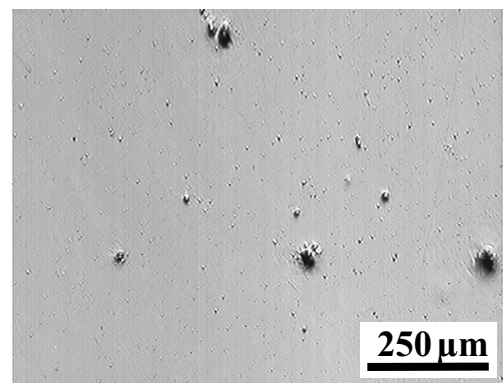

(b) Flowing with bubble $\left(A_{e} / A_{0}=0.02\right)$

Figure 9: Effect of micro bubbles on cavitation damage after $10^{4}$ impacts in the MIMTM. 


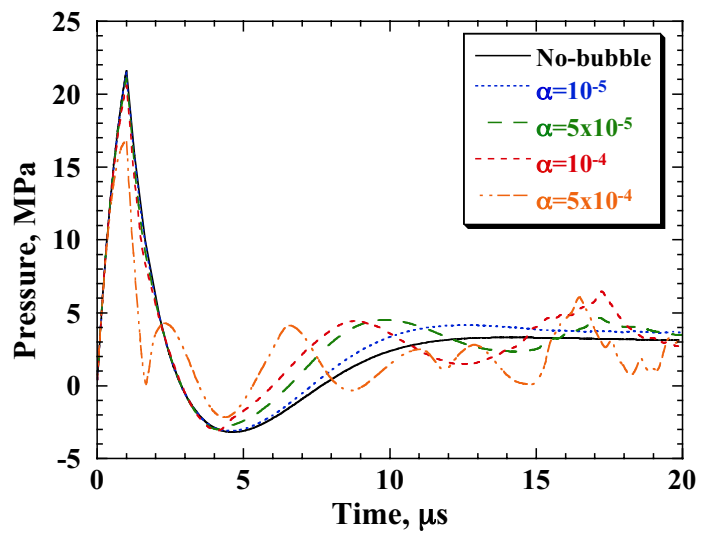

Figure 10: Pressure time-response in bubbly liquid mercury with $50 \mu \mathrm{m}$ gas-bubbles at various void fractions.

vestigated by using the Keller equation Eq. (3), where $P_{e x}$ is the pressure timeresponse shown in Fig. 10. Figure 11 shows the cavitation bubble growth behaviors, which clearly depend on the void fraction. It is noted that the core of the cavitation bubble, initial bubble radius, was defined as $1 \mu \mathrm{m}$. The bubbles grow up to the maximum radius in less than $10 \mu \mathrm{s}$ after the peak negative pressure appearing, i.e. the time delay due to inertia and viscous effects is recognized. The negative pressure period seems a critical factor for the cavitation bubble size. The cavitation growth in microscopic time-scale is certainly suppressed by the injected gas-bubbles. The cavitation intensity $I_{C}$, i.e. acoustic pressure injected during collapsing process, is associated with the third power of the maximum expansion ratio, $\left(R_{\max } / R_{0}\right)^{3}[16]$, as given by

$$
I_{C}=f\left(\left(\frac{R_{\max }}{R_{0}}\right)^{3}\right)
$$

In fact, it was recognized that the incubation period of mercury cavitation erosion is linearly related with the third power of cavitation bubble size [17]. Therefore, the cavitation damage might be mitigated by injected gas-bubbles even in the mi- 
croscopic time-scale.

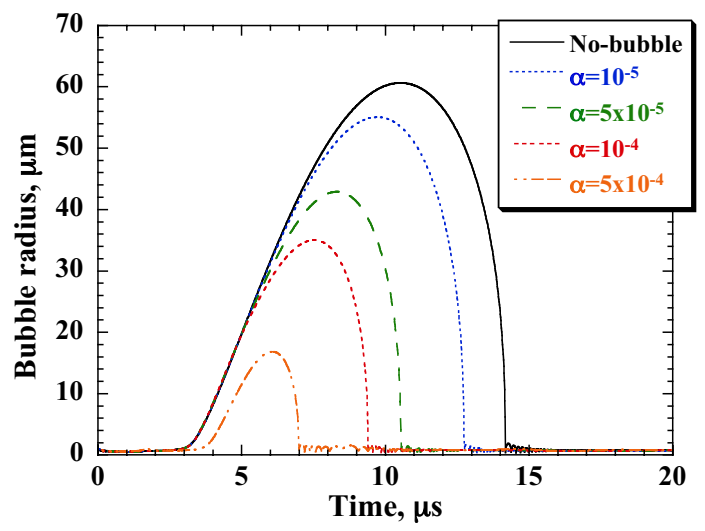

Figure 11: Cavitation bubble growth behavior for pressure time response shown in Fig. 10. $\left(R_{0}=1\right.$ $\mu \mathrm{m})$

\section{Discussion}

As for the mercury target for the spallation neutron source, in the microscopic time-scale, $\mu$ s scale, the compressive pressure waves induced by the proton beam bombarding in mercury propagates outward to the target vessel and reflects with the phase changing to the negative pressure due to the impedance difference between the vessel wall and the mercury, and propagates inward. After that, in the macroscopic time- scale, ms scale, alternate pressure changes result from the interaction with structural vessel vibrations through the propagation. In the former the strong compressive and relatively high negative pressures are induced in a few $\mu \mathrm{s}$, and in the latter low negative pressures impose continuously for a few ms. The possibility of the cavitation inception is present in both cases. The cavitation damage determines the target lifetime and is the most crucial issue to increase 
the proton beam power. In order to mitigate the cavitation damage, therefore, the gas-bubble will be injected into the flowing mercury.

One considers whether the gas-bubbles themselves will work as the violent cavitation bubbles or not. Figure 12 shows the expansion ratio $R / R_{0}$ in the macroscopic time-scales, which are calculated by using Eq.3 with $R_{0}$ of 50, 100, $500 \mu \mathrm{m}$ and the pressure responses given in Fig. 6 . The $R / R_{0}$ is likely to be associated with acoustic pressure generated at collapsing. In the case of $R_{0}<10 \mu \mathrm{m}$, the bubble growth was not observed as shown in Fig. 7 in the macroscopic time-scales, because the magnitude of negative pressure was reduced by the gas-bubble injection so that the pressure get to be beyond the tensile threshold for bubble growth.

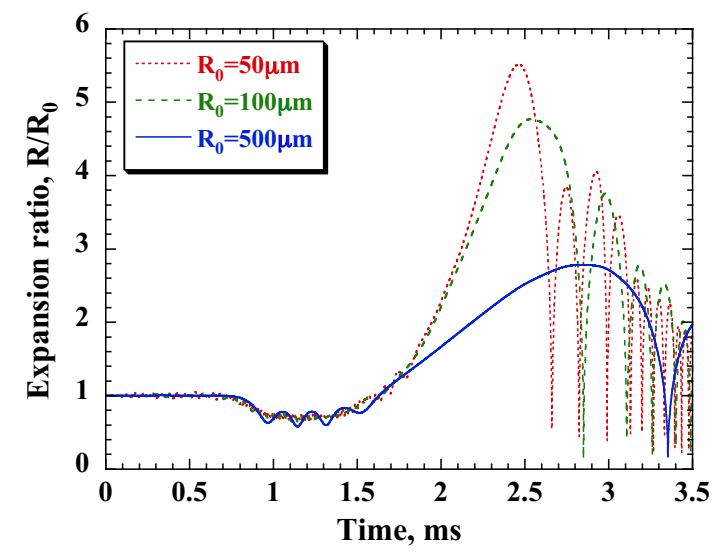

Figure 12: Expansion ratio in macroscopic time-scale.

Figure 13 shows $R / R_{0}$ in the microscopic time-scale at $R_{0}=50 \mu \mathrm{m}$ for gasbubbles and $R_{0}=1$ and $0.5 \mu \mathrm{m}$ for cavitation bubbles under gas-bubble injection with void fraction $10^{-4}$ at $50 \mu \mathrm{m}$ in radius. It was deduced from Figs. 12 and 13 that the $R / R_{0}$ for cavitation in the microscopic time-scale is clearly much larger than those of gas-bubbles and the cavitation caused in the microscopic time-scale is likely to be the most dangerous, in particular, much the worse as taking ac- 
count of radiation induced cavitation [18]. A neutron-nucleus interaction might result in the nucleation and the growth of tiny bubbles leading to the cavitation phenomenon in microscopic time-scale. That is, the negative pressure is strong enough for such tiny bubbles to grow up, even if the gas-bubbles are injected.

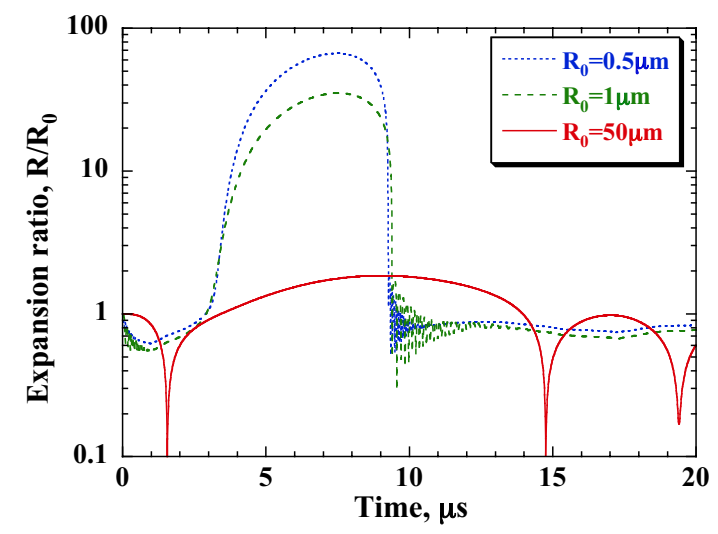

Figure 13: Expansion ratio in microscopic time-scale.

\section{Conclusion}

Mercury cavitation gets to be a crucial issue to increase the power in the mercury target for pulsed spallation neutron sources. The gas-bubble injection into the mercury will be applied to mitigate the pressure wave that induces the cavitation phenomenon. The injected gas-bubbles will be workable well to suppress the cavitation inception in the macroscopic time-scale because the magnitude of negative pressure is raised by the bubble injection beyond the tensile threshold for the cavitation inception. In the microscopic time- scale, the gas-bubbles certainly mitigate the cavitation intensity, but not sufficiently: i.e. in other words, the possibility of the cavitation inception is present in mercury with injected gas-bubbles. 


\section{Acknowledgements}

This work was partly supported by the Japan Society for the Promotion of Sci-

ence through a Grant-in-Aid for Scientific Research (Nos. 20360090 and 23360088).

\section{References}

[1] Y. Ikeda, J-PARC status update, Nucl. Instrum. Methods Phys. Res., Sect. A 600 (2009) 1-4.

[2] T. McManamy, SNS October 2008 status, J. Nucl. Mater, 398 (2010) 10-14.

[3] K. Skala, G.S. Bauer, On the pressure wave problem in liquid metal targets, in: Proc. 8th Int. Collabo. on Adv. Neutron Source (ICANS-XIII), Villigen, Switzerland, October 11-14, 1995, pp. 559-576.

[4] M. Futakawa, K. Kikuchi, H. Conrad, H. Stechemesser, Pressure and stress waves in a spallation neutron source mercury target generated by high-power proton pulses, Nucl. Instrum. Methods Phys. Res., Sect. A 439 (2000) 1-7.

[5] J. R. Haines, B. W. Riemer, D. K. Felde, J. D. Hunn, S. J. Pawel, C. C. Tsai, Summary of cavitation erosion investigations for the SNS mercury target, J. Nucl. Mater., 343 (2005) 58-69.

[6] M. Futakawa, T. Naoe, H. Kogawa, C.C. Tsai, Y. Ikeda, Pitting damage formation up to over 10 million cycles -off-line test by MIMTM-, J. Nucl. Sci. Techol., 40 (2003) 895-904.

[7] M. Futakawa, T. Naoe, C.C. Tsai, H. Kogawa, S. Ishikura, Y. Ikeda, H. Soyama, H. Date, Pitting damage by pressure waves in a mercury target, J. Nucl. Mater. 343 (2005) 70-80. 
[8] L.K. Mansur, Materials research and development for the spallation neutron source mercury target, J. Nucl. Mater. 318 (2003) 12-25.

[9] T. Naoe, M. Futakawa, T. Shoubu, T. Wakui, H. Kogawa, H. Takeuchi, M. Kawai, Mitigation technologies for damage induced by pressure waves in high-power mercury spallation neutron sources (I) -Material surface improvement-, J. Nucl. Sci. Technol., 45 (2008) 698-703.

[10] K. Okita, S. Takagi, Y. Matsumoto, Propagation of pressure waves, caused by a thermal shock, in liquid metals containing gas bubbles, J. Fluid Sci. Technol., 3 (2008) 116-128.

[11] H. Kogawa, H. Haga, T. Naoe, H. Kinoshita, M. Ida, M. Futakawa, Development of bubble injection technique in JSNS mercury target, Proceedings of 19th meeting on Collaboration of Advanced Neutron Sources (ICANS XIX), 8th-12th March (2010), Grindelwald, Switzerland.

[12] L. Ni, G. S. Bauer, H. Spitzer, Effects of pulsed power into a liquid metal target, Nucl. Instrum. Methods Phys. Res., Sect. A 425 (1999) 57-64.

[13] T. Sato, K. Niita, N. Matsuda, S. Hashimoto, Y. Iwamoto, S. Noda, T. Ogawa, H. Iwase, H. Nakashima, T. Fukahori, K. Okumura, T. Kai, S. Chiba, T. Furuta and L. Sihver, Particle and Heavy Ion Transport Code System PHITS, Version 2.52, J. Nucl. Sci. Technol., 50 (2013) 913-923.

[14] H. Kogawa, S. Hasegawa, M. Futakawa, B. Riemer, M. Wendel, J. Haines, Numerical study on pressure wave propagation in a mercury loop, J. Nucl. Mater., 377 (2008) 195-200. 
[15] M. Futakawa, H. Kogawa, S. Hasegawa, T. Naoe, M. Ida, K. Haga, T. Wakui, N. Tanakan, Y. Matsumoto, Y. Ikeda, Mitigation Technologies for Damage Induced by Pressure Waves in High-Power Mercury Spallation Neutron Sources (II) -Bubbling Effect to Reduce Pressure Wave-, J. Nucl. Sci. Technol., 45 (2008) 1041-1048.

[16] e.g. C. E. Brennen, Cavitation and Bubble Dynamics, Oxford University Press, New York, 1995.

[17] M. Futakawa, T. Naoe, H. Kogawa, T. Wakui, K. Haga, Mercury cavitation and its mitigation technique for high power sulse spallation neutron sources, Proceedings of WIMRC 3rd International Cavitation Forum 2011, 4th-6th July (2011), University of Warwick, UK.

[18] D. Lieberman, Radiation-induced cavitation, Phys. Fluids, 2 (1959) 466468. 